\title{
Treatment of HER2-Positive Metastatic Breast Cancer
}

\author{
Sandra M. Swain, M.D., \\ MedStar Washington Hospital Center, Washington, DC \\ Emma Clark, M.Sc., and \\ Roche Products, Welwyn Garden City, United Kingdom \\ José Baselga, M.D., Ph.D. \\ Memorial Sloan Kettering Cancer Center, New York, NY \\ Sandra M. Swain: sandra.m.swain@medstar.net
}

\section{The Authors Reply}

In response to van Ramshorst and Sonke regarding outcomes in the subgroup of 88 patients with prior trastuzumab treatment for early breast cancer: the hazard ratio for death from any cause favored the pertuzumab group (in which patients received pertuzumab, trastuzumab, and docetaxel; hazard ratio, $0.80 ; 95 \%$ confidence interval, 0.44 to 1.47 ); owing to the small number of patients, the confidence intervals were wide. The median overall survival was 46.6 months among 41 patients in the control group (in which patients received placebo, trastuzumab, and docetaxel) and 53.8 months among 47 patients in the pertuzumab group.

Although the proportion of patients with prior trastuzumab treatment was lower in our study than in current clinical practice, historical context is important. Study recruitment began less than 2 years after the initial approval of adjuvant trastuzumab. Ongoing improvements in early breast cancer therapy have lowered recurrence rates and increased the proportion of patients with new metastatic breast cancer. ${ }^{1}$ The observational trial registHER ${ }^{2}$ recruited patients before the widespread use of trastuzumab in early breast cancer and reported fewer patients with new metastatic breast cancer than the more recent Systemic Therapies for HER2-Positive Metastatic Breast Cancer Registry (SystHERs) trial ${ }^{1}$ (32.0\% vs. 49.2\%). These findings emphasize the relevance of our study data to current clinical practice.

\section{References}

1. Tripathy, D.; Brufsky, A.; Cobleigh, M., et al. Increasing proportion of de novo compared with recurrent HER2-positive metastatic breast cancer: early results from the Systemic therapies for HER2-positive metastatic breast cancer registry study. Presented at the 37th Annual San Antonio Breast Cancer Symposium; San Antonio, TX. December 9-13 2014; abstract

2. Yardley DA, Kaufman PA, Brufsky A, et al. Treatment patterns and clinical outcomes for patients with de novo versus recurrent HER2-positive metastatic breast cancer. Breast Cancer Res Treat. 2014; 145:725-34. [PubMed: 24706168] 